\title{
Development of a model on factors affecting instrumental activities of daily living in people with mild cognitive impairment - a Delphi study
}

\author{
Marina Bruderer-Hofstetter ${ }^{1,2^{*}}$ (D), Sietske A. M. Sikkes ${ }^{3}$, Thomas Münzer ${ }^{4,5}$ and Karin Niedermann ${ }^{1}$
}

\begin{abstract}
Introduction: The level of function of instrumental activities of daily living (IADL) is crucial for a person's autonomy. A clear understanding of the nature of IADL and its limitations in people with mild cognitive impairment (MCI) is lacking. Literature suggests numerous possible influencing factors, e.g. cognitive function, but has not considered other domains of human functioning, such as environmental factors. Our aim was to develop a comprehensive model of IADL functioning that depicts the relevant influencing factors.

Methods: We conducted a four-round online Delphi study with a sample of international IADL experts $(N=69)$. In the first round, panelists were asked to mention all possible relevant cognitive and physical function factors, as well as environmental and personal factors, that influence IADL functioning. In the subsequent rounds, panelists rated the relevance of these factors. Consensus was defined as: 1) $\geq 70 \%$ agreement between panelists on a factor, and 2) stability over two successive rounds.

Results: Response rates from the four rounds were high (83 to 100\%). In the first round, 229 influencing factors were mentioned, whereof 13 factors reached consensus in the subsequent rounds. These consensual factors were used to build a model of IADL functioning. The final model included: five cognitive function factors (i.e. memory, attention, executive function, and two executive function subdomains -problem solving / reasoning and organization / planning); five physical function factors (i.e. seeing functions, hearing functions, balance, gait / mobility functions and functional mobility functions); two environmental factors (i.e. social network / environment and support of social network / environment); and one personal factor (i.e. education).

(Continued on next page)
\end{abstract}

\footnotetext{
* Correspondence: marina.bruderer-hofstetter@zhaw.ch

'School of Helath Professions, Institute of Physiotherapy, Zurich University of Applied Sciences, Winterthur, Switzerland

2Department of Health Sciences and Health Policy, University of Lucerne, Lucerne, Switzerland

Full list of author information is available at the end of the article
}

(c) The Author(s). 2020 Open Access This article is licensed under a Creative Commons Attribution 4.0 International License, which permits use, sharing, adaptation, distribution and reproduction in any medium or format, as long as you give appropriate credit to the original author(s) and the source, provide a link to the Creative Commons licence, and indicate if changes were made. The images or other third party material in this article are included in the article's Creative Commons licence, unless indicated otherwise in a credit line to the material. If material is not included in the article's Creative Commons licence and your intended use is not permitted by statutory regulation or exceeds the permitted use, you will need to obtain permission directly from the copyright holder. To view a copy of this licence, visit http://creativecommons.org/licenses/by/4.0/ The Creative Commons Public Domain Dedication waiver (http://creativecommons.org/publicdomain/zero/1.0/) applies to the data made available in this article, unless otherwise stated in a credit line to the data. 
(Continued from previous page)

Conclusions: This study proposes a comprehensive model of IADL functioning in people with $\mathrm{MCl}$. The results from this Delphi study suggest that IADL functioning is not merely affected by cognitive function factors, but also by physical function factors, environmental factors and personal factors. The multiplicity of factors mentioned in the first round also underlines the individuality of IADL functioning in people with $\mathrm{MCl}$. This model may serve as a basis for future research in IADL functioning in people with $\mathrm{MCl}$.

Keywords: Instrumental activities of daily living (IADL), Mild cognitive impairment (MCI), Model, Delphi study, Physical function, Cognitive function, Environmental factors, Personal factors

\section{Introduction}

Instrumental activities of daily living (IADL) are complex tasks, such as managing finances or performing a shopping task [1]. Within the context of cognitive decline, IADLs have been defined as intentional and complex everyday activities for which multiple cognitive processes are necessary, particularly high-level controlled processes' [2]. Preserved IADL abilities allow people to live independently and to maintain their autonomy. They are crucial on the individual and the societal level [3]. Performance of IADLs are related to an appropriate physical health [4] and cognitive function [3], with IADL limitations being associated with reduced wellbeing [5] and increased caregiver burden, supervision time and total societal costs [6]. Cognitive impairments affect IADL performance [7-9].

Mild cognitive impairment (MCI) is defined as a transient state between normal cognitive ageing and early dementia and is primarily characterized by loss of cognitive function in one or more cognitive domains, but with preserved functional abilities [10]. However, IADL limitations might be present at the MCI state $[9,11,12]$. A recent meta-analysis demonstrated that people with MCI had greater IADL limitations compared to healthy controls, with an effect size of $g=0.76$ [12]. Furthermore, IADL limitations were found to discriminate people with $\mathrm{MCI}$ from people with normal cognition [13] and predict conversion to dementia [14, 15]. These findings may have led to the incorporation of IADL difficulties into the current diagnostic criteria of mild neurocognitive disorder (incorporating MCI) [16].

IADL limitations in people with MCI are associated with cognitive impairment [5, 12]. Empirical data [7] and a meta-analysis [17] estimated that 20 and 23\%, respectively, of IADL variability was due to cognition. This implies that other factors also seem to be important in influencing IADL performance. Several studies reported that people with MCI have difficulties in motor function [18-20] and clinical measures, incorporating muscular strength, cardiovascular function and physical activity, predicted a decrease in cognitive function after 1 year [21]. Thus, a clear understanding of the nature of IADL limitations in people with MCI is lacking.
The international classification of functioning, disability and health (ICF) provides a framework for the description of human functioning [22]. The framework considers functioning and disability as outcomes resulting from a health condition, as well as environmental and personal factors and, therefore, may allow a mapping of IADL performance in people with MCI [22]. To date, evidence suggests that there are several physical $[18,20,23-26]$ and cognitive function factors $[5,7,8,17]$, as well as personal [27-29] and environmental factors [30-32] influencing IADL performance in people with MCI. However, to our knowledge, previous studies have investigated only a limited number of possible influencing factors, e.g. the association between factors of cognitive function and IADL performance, without $[5,8,17]$ or with limited consideration of factors from other domains of the ICF, i.e. factors of physical function, environmental and personal factors [7, 27]. We have, therefore, taken a different approach to modelling the complexity of factors influencing IADL performance. The aim of this study was to develop a model of the physical and cognitive function factors, environmental factors and personal factors contributing to IADL performance in people with $\mathrm{MCI}$, by means of a multiple-round Delphi study based on consensus from an expert panel.

\section{Methods \\ Study design}

Between October 2018 and April 2019, a Delphi study was conducted with an international panel of IADL experts (panelists). This design aims to seek consensus of the opinions of a group of panelists through a series of structured questionnaires, i.e. rounds with controlled feedback. Anonymity between panelists is another key element of the study [33]. Two different concepts of consensus were assessed: agreement and stability [34]. Agreement was defined twofold: $\geq 70 \%$ or $\leq 10 \%$ of all panelists rate a factor as relevant. Stability determines the consistency of responses and was defined as $<15 \%$ difference in percent-agreement between two succeeding rounds [35]. This was used as a measure to stop the Delphi study [36]. The maximum number of rounds was set at four, including the option to omit the fourth round 
when stability between the second and third rounds was achieved [36].

\section{Selection of panelists}

A selective sampling procedure was used to define the panel [37]. International researchers with authorship of relevant research articles [38] were identified based on a literature search performed in Medline and Web of Science. The search resulted in a total of 163 potential panelists. The panel sample was complemented with researchers from personal networks (SAMS, TM) and, to achieve a broader spectrum in the panel [37], clinicians, neuropsychologists and health professionals who worked with people with MCI on a daily basis in Memory Clinics in the eastern part of Switzerland. The latter were invited by email. Panelists were free to forward the invitation email to whomever they considered as relevant (snowball sampling) [38]. Sixty-nine panelists $(N=69)$ agreed to participate in the Delphi study.

\section{Procedure}

Online questionnaires were pretested and implemented in an EFS (Enterprise Feedback Suite) survey (version 18.3 Questback / Unipark) and distributed by email. In each round, non-responders received a first reminder after 2 weeks and a second reminder 2 weeks later. Questionnaires in subsequent rounds were sent to all panelists who had responded to the questionnaire of the preceding round. Missing data in questionnaires were excluded from data analysis.

\section{First-round questionnaire and analysis}

In the first round, personal details of the panelists (i.e. country of residence, professional background, current occupation / position and years of experience) were collected.

The questionnaire described the aim of the Delphi study, a short summary of current knowledge and the definition of IADL in accordance with Sikkes and Rotrou [2]. The ICF framework [22] was provided as a model for further discussion. The first-round questionnaire asked one open-ended question: "What are the relevant factors of physical and cognitive function, as well as, personal and environmental factors influencing IADL functioning in people with MCI?" Panelists were prompted to list all relevant factors for each domain separately (i.e. physical function, cognitive function, environmental factors and personal factors). The first-round questionnaire can be found in the Additional file 1.

A deductive content analysis was performed on all responses [39]: two researchers ( $\mathrm{MB}$ and a research fellow) independently grouped the mentioned factors into the domains of the ICF framework [22]. Accordingly, environmental factors were defined as factors that are not under the control of the person and personal factors as those possible influencing factors independent of MCI. Depending on personal preference, some factors could be seen both as "personal factors" as well as "environmental factors", e.g. socio-cultural factors. If appropriate, these factors were included in both domains. Answers describing the same factor in a slightly different manner were merged into one factor [38], whereas specificallynamed factors were not comprised into broader functions, e.g. "planning" into "executive function". Factors were formulated neutrally, without using qualifiers [22], e.g. "impaired vision" was formulated as "seeing function". Differences in categorization were resolved through discussion with a third researcher (KN) [38].

\section{Second-round questionnaire and analysis}

The questionnaire included all factors mentioned in the first round, together with their frequency, presented for each domain separately. Panelists were then asked to state whether the presented factors were relevant or not.

Percent-agreement on the factors was calculated. Factors reaching $\geq 70 \%$ or $\leq 10 \%$ agreement were excluded from the third round questionnaire in accordance with the Delphi methodology [38]. Factors reaching $\geq 70 \%$ percent-agreement were included in the model.

\section{Third-round questionnaire and analysis}

The questionnaire included all factors with a percentagreement of $\geq 10 \%$ and $\leq 70 \%$, including their frequency and percent-agreement. In addition, a first draft of the model was presented. Panelists were asked to rerate the relevance of these factors. Agreement on the factors was calculated and stability between the second and third rounds was assessed.

\section{Fourth-round questionnaire and analysis}

The second draft of the model was presented. Panelists were asked to provide their feedback on the model and to state whether it was consistent with their conception of IADL performance in people with MCI. Panelists were further asked to rerate on the 10 factors that had not reached consensus or stability in the third round [33].

The feedbacks on the second draft of the model were analyzed using inductive content analysis [39]. Accordingly, one researcher (MB) coded all individual panelists' responses into categories using a stepwise procedure; frequencies of categories were counted. Percent-agreement on the model was calculated, as well as stability and consensus on the remaining factors. If a factor reached stability and consensus, this factor was included in the final model. 


\section{Results}

\section{Results first round}

Sixty panelists (87\% response rate; 60 / 69) completed the first-round questionnaire. Panelists (64\% female) were from Europe (62\%), North and South America (32\%) and Australia (6\%). Half of the panelists were currently working in academia or research and the other half in the clinical field. Details of professional background and current occupation / position are presented in Table 1. Of all the panelists: 20 (34\%) had more than 20 years of experience within their respective field; 15 (25\%) between 11 and 20 years; 21 (36\%) between five and 10 years; 3 (5\%) less than 5 years; one panelist did not provide this information.

A total of 229 factors were mentioned in the first round, of which 42 (18\%) were physical function factors,

Table 1 Panel professional background and experience

\begin{tabular}{lll}
\hline Professional background & Specialization & $\mathrm{n}$ \\
\hline Physician & & 17 \\
Psychologist & 9 \\
Neuropsychologist & 5 \\
Psychopharmacologist & & 1 \\
Epidemiologist & & 1 \\
Physical therapist & & 7 \\
Occupational therapist & & 9 \\
Nurse & & 9 \\
Not stated & Geriatrics / Gerontology & 6 \\
& Neurology & 4 \\
& (Geriatric) Psychiatry & 6 \\
& Epidemiology & 2 \\
& Anthropology & 1 \\
& Research (i.e. PhD) & 12
\end{tabular}

\section{Current occupation}

$\begin{array}{ll}\text { Chair / Dean } & 3 \\ \text { Professor (assoc. / asst.) } & 10 \\ \text { Lecturer } & 4 \\ \text { Researcher } & 13 \\ \text { Head of department (i.e. memory clinic) } & 7 \\ \text { Practicing physician } & 8 \\ \text { Clinical (neuro) - psychologist } & 10 \\ \text { Dementia specialist } & 2 \\ \text { Physical therapist } & 3 \\ \text { Occupational therapist } & 4 \\ \text { Nursing } & 7 \\ \text { Professor emeritus / retired } & 2 \\ \text { Not stated } & 1\end{array}$

Current occupation multiple naming possible; $\mathrm{n}=$ absolute frequency
48 (21\%) cognitive function factors, 57 (25\%) environmental factors and $82(36 \%)$ personal factors, with frequencies ranging from one to 24 (Table 2).

\section{Results second round}

Fifty-three panelists (88\% response rate; 53 / 60) completed the second-round questionnaire. One questionnaire was excluded in data analysis due to missing data, in two questionnaires data was missing for cognitive function, environmental and personal factors and in one questionnaire data was missing for environmental and personal factors. The panel reached consensus on 126 factors (55\%). Nine of these factors were rated as relevant by $\geq 70 \%$ of panelists and were included in the model, whilst 117 (51\%) factors were rated as relevant by $\leq 10 \%$ and were subsequently excluded from the third round (Table 2). Overall, 103 (45\%) factors did not reach consensus in the second round and were included in the third-round questionnaire (Table 2).

\section{Results third round}

Fifty-three panelists (100\% response rate; 53 / 53) completed the third-round questionnaire. Two questionnaires were excluded from data analysis due to missing data. Of the remaining 103 factors, two (2\%) reached consensus and were included in the model. Stability of responses between the second and third round was ascertained for 93 (90\%) factors. Ten factors (10\%) did not reach stability and were therefore included in the fourth-round questionnaire.

\section{Results fourth round}

Forty-four panelists (83\% response rate; 44 / 53) responded to the fourth-round questionnaire. Thirtythree (62\%; 33 / 53) panelists provided feedback on the model with $28(85 \%)$ stating that the model met their conception of IADL functioning in people with MCI. Feedback on the model covered: factors not included in the model; lack of weighting and relatedness of the factors; one panelist questioned the method itself (Table 3).

The two additional factors reached consensus and stability between the third and fourth rounds and were consequently included in the final model (Fig. 1). Stability between the third and fourth rounds was not reached for five factors (50\%), i.e. judgment / decision making, working memory, language functions, financial situation and experience / familiarity with certain IADL tasks (Table 2).

\section{Discussion}

The results of this Delphi study illustrate how panelists from the academic / research and clinical practice perspectives agreed on several factors of cognitive and physical functions, as well as personal factors and 
Table 2 Mentioned factors

\begin{tabular}{|c|c|c|c|c|c|c|c|c|c|}
\hline \multicolumn{10}{|l|}{ Physical function factors } \\
\hline & $\begin{array}{l}\text { First } \\
\text { round }\end{array}$ & $\begin{array}{l}\text { Second } \\
\text { round }\end{array}$ & $\%$ & $\begin{array}{l}\text { Third } \\
\text { round }\end{array}$ & $\%$ & $\begin{array}{l}\% \\
\text { diff. }\end{array}$ & $\begin{array}{l}\text { Fourth } \\
\text { round }\end{array}$ & $\%$ & $\begin{array}{l}\% \\
\text { diff. }\end{array}$ \\
\hline${ }^{a}$ vision / seeing functions & 13 & 39 & 75 & & & & & & \\
\hline vision acuity & 4 & 6 & 11.5 & 6 & 11.8 & 0.2 & & & \\
\hline eye movement functions & 1 & 0 & & & & & & & \\
\hline ahearing functions & 18 & 37 & 71.2 & & & & & & \\
\hline sensory functions & 2 & 7 & 13.5 & 7 & 13.7 & 0.3 & & & \\
\hline proprioceptive functions & 1 & 6 & 11.5 & 5 & 9.8 & -1.7 & & & \\
\hline touch functions & 3 & 6 & 11.5 & 3 & 5.88 & -5.7 & & & \\
\hline smell functions & 1 & 1 & 1.9 & & & & & & \\
\hline pain & 5 & 19 & 36.5 & 24 & 47.1 & 10.5 & & & \\
\hline vestibular functions & 3 & 8 & 15.4 & 8 & 15.7 & 0.3 & & & \\
\hline vestibular function of balance & 1 & 2 & 3.8 & & & & & & \\
\hline stability & 2 & 3 & 5.8 & & & & & & \\
\hline abalance & 20 & 37 & 71.2 & & & & & & \\
\hline${ }^{a}$ mobility / gait functions & 17 & 38 & 73.1 & & & & & & \\
\hline fall risk / fall experience & 2 & 10 & 19.2 & 16 & 31.4 & 12.1 & & & \\
\hline walking speed & 3 & 4 & 7.7 & & & & & & \\
\hline$c_{\text {functional mobility (e.g. stair climbing) }}$ & 4 & 22 & 42.3 & 32 & 62.7 & 20.4 & 31 & 79.5 & -16.7 \\
\hline ability to travel & 3 & 4 & 7.7 & & & & & & \\
\hline general physical endurance functions & 16 & 26 & 50 & 19 & 37.3 & -12.7 & & & \\
\hline aerobic capacity & 1 & 1 & 1.9 & & & & & & \\
\hline fatigability & 3 & 10 & 19.2 & 7 & 13.7 & -5.5 & & & \\
\hline muscle power functions (general physical strength) & 15 & 30 & 57.7 & 33 & 64.7 & 7 & & & \\
\hline lower limb power (lower extremity strength) & 4 & 2 & 3.8 & & & & & & \\
\hline grip strength & 5 & 7 & 13.5 & 11 & 21.6 & 8.1 & & & \\
\hline upper extremity strength & 1 & 1 & 1.9 & & & & & & \\
\hline manual dexterity (fine motor skills) & 9 & 25 & 48.1 & 27 & 52.9 & 4.9 & & & \\
\hline fine motor coordination & 5 & 12 & 23.1 & 15 & 29.4 & 6.3 & & & \\
\hline coordination & 4 & 9 & 17.3 & 11 & 21.6 & 4.3 & & & \\
\hline control of body movement functions & 1 & 2 & 3.8 & & & & & & \\
\hline visuo-motor coordination capacity & 1 & 8 & 15.4 & 16 & 31.4 & 16 & 13 & 33.3 & 2.0 \\
\hline tremor & 1 & 0 & & & & & & & \\
\hline mobility of joints functions (e.g. range of motion) & 13 & 18 & 34.6 & 8 & 15.7 & -18.9 & 6 & 15.4 & -0.3 \\
\hline mobility of the spine and cervical spine & 1 & 0 & & & & & & & \\
\hline gross motor function & 3 & 6 & 11.5 & 3 & 5.88 & -5.7 & & & \\
\hline motor speed & 5 & 11 & 21.2 & 14 & 27.5 & 6.3 & & & \\
\hline agility & 1 & 0 & & & & & & & \\
\hline functional reach & 1 & 4 & 7.7 & & & & & & \\
\hline functions of the cardiovascular system & 2 & 5 & 9.6 & & & & & & \\
\hline cardiorespiratory reserve & 1 & 1 & 1.9 & & & & & & \\
\hline blood pressure & 1 & 1 & 1.9 & & & & & & \\
\hline cholesterol values & 1 & 1 & 1.9 & & & & & & \\
\hline Respiratory functions & 2 & 2 & 3.8 & & & & & & \\
\hline
\end{tabular}


Table 2 Mentioned factors (Continued)

\begin{tabular}{|c|c|c|c|c|c|c|c|c|c|}
\hline \multicolumn{10}{|l|}{ Cognitive function factors } \\
\hline & $\begin{array}{l}\text { First } \\
\text { round }\end{array}$ & $\begin{array}{l}\text { Second } \\
\text { round }\end{array}$ & $\%$ & $\begin{array}{l}\text { Third } \\
\text { round }\end{array}$ & $\%$ & $\begin{array}{l}\% \\
\text { diff. }\end{array}$ & $\begin{array}{l}\text { Fourth } \\
\text { round }\end{array}$ & $\%$ & $\begin{array}{l}\% \\
\text { diff. }\end{array}$ \\
\hline aattention functions & 23 & 39 & 78 & & & & & & \\
\hline sustaining attention & 9 & 2 & 4 & & & & & & \\
\hline shifting attention & 5 & 4 & 8 & & & & & & \\
\hline dividing attention & 6 & 7 & 14 & 10 & 19.6 & 5.6 & & & \\
\hline sharing attention & 2 & 2 & 4 & & & & & & \\
\hline processing speed functions & 7 & 19 & 38 & 17 & 33.3 & -4.7 & & & \\
\hline reaction time & 2 & 4 & 8 & & & & & & \\
\hline a executive functions & 24 & 39 & 78 & & & & & & \\
\hline sequencing & 4 & 2 & 4 & & & & & & \\
\hline borganization and planning & 11 & 27 & 54 & 37 & 72.5 & 18.5 & & & \\
\hline cognitive / mental flexibility & 7 & 22 & 44 & 22 & 43.1 & -0.9 & & & \\
\hline insight & 9 & 2 & 4 & & & & & & \\
\hline judgement / decision making & 5 & 14 & 28 & 22 & 43.1 & 15.1 & 25 & 64.1 & 21.0 \\
\hline b problem solving / reasoning & 8 & 27 & 54 & 36 & 70.6 & 16.6 & & & \\
\hline inhibition & 3 & 2 & 4 & & & & & & \\
\hline initiation & 1 & 1 & 2 & & & & & & \\
\hline${ }^{a}$ memory functions & 25 & 42 & 84 & & & & & & \\
\hline learning & 4 & 3 & 6 & & & & & & \\
\hline short-term memory & 5 & 3 & 6 & & & & & & \\
\hline long-term memory & 3 & 0 & & & & & & & \\
\hline episodic memory & 3 & 2 & 4 & & & & & & \\
\hline semantic memory & 1 & 0 & & & & & & & \\
\hline working memory & 7 & 9 & 18 & 18 & 35.3 & 17.3 & 24 & 61.5 & 26.2 \\
\hline prospective memory & 2 & 3 & 6 & & & & & & \\
\hline retrieval and processing of memory & 7 & 4 & 8 & & & & & & \\
\hline language functions & 13 & 33 & 66 & 25 & 49.0 & -17 & 26 & 66.7 & 17.6 \\
\hline language comprehension (written and spoken) & 7 & 11 & 22 & 10 & 19.6 & -2.4 & & & \\
\hline semantic fluency & 4 & 1 & 2 & & & & & & \\
\hline semantic knowledge & 1 & 0 & & & & & & & \\
\hline language execution & 3 & 1 & 2 & & & & & & \\
\hline word finding & 3 & 3 & 6 & & & & & & \\
\hline calculation functions & 9 & 4 & 8 & & & & & & \\
\hline abstraction & 1 & 0 & & & & & & & \\
\hline perceptual functions & 8 & 12 & 24 & 7 & 13.7 & -10.3 & & & \\
\hline perceptual-motor functions & 1 & 1 & 2 & & & & & & \\
\hline visuo-spatial functions & 9 & 21 & 42 & 22 & 43.1 & 1.1 & & & \\
\hline visuo-perceptual functions & 5 & 2 & 4 & & & & & & \\
\hline psychomotor functions & 5 & 6 & 12 & 6 & 11.8 & -0.2 & & & \\
\hline orientation & 5 & 11 & 22 & 17 & 33.3 & 11.3 & & & \\
\hline energy and drive / stamina & 2 & 3 & 6 & & & & & & \\
\hline metacognition & 3 & 3 & 6 & & & & & & \\
\hline motivation & 16 & 29 & 58 & 28 & 54.9 & -3.1 & & & \\
\hline
\end{tabular}


Table 2 Mentioned factors (Continued)

mood
alertness / vigilance
awareness
intelligence
social cognition
emotional functions

9
5
1
3
8
8

$\begin{array}{lllll}12 & 24 & 13 & 25.5 & 1.5 \\ 7 & 14 & 12 & 23.5 & 9.5 \\ 3 & 6 & & & \\ 1 & 2 & & & \\ 15 & 30 & 15 & 29.4 & -0.6 \\ 13 & 26 & 10 & 19.6 & -6.4\end{array}$

Environmental factors

parental beliefs

round

Second

round

societal attitudes

social expectations

social norms

socio-cultural factors

widowed / changes in personal network

family support

${ }^{a}$ social network / social environment

cnetwork / social support

loneliness / isolation

personal assistance available

immediate family (e.g. children, siblings)

extended family (e.g. spouse)

weather

climate

extreme temperatures

noise

adequate light

air quality / pollution

place of residence (rural versus urban environment)

neighborhood

age-friendliness of environment

environmental demands

familiarity with environment

challenging environment

physical environment / living environment

presence of gangs

type of house / apartment

adaptation / age-friendliness / safety of home environment

Housing / immediate home environment

accessibility of the house / apartment

living form

living with family / family nearby

living situation (independent / dependent)

financial situation / resources

financial resources for dental care
2

4

3

4

4

3

3

13

11

5

3

6

4

1

3

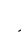

1

3

1

3

7

4

1

3

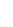

1

3

6

1

3

7

7

9

1

5

3

1

13

1

\section{0}

6

$$
5
$$$$
11
$$$$
19
$$$$
7
$$$$
11
$$$$
35
$$$$
35
$$$$
16
$$$$
20
$$$$
4
$$

$$
9
$$$$
6
$$$$
1
$$$$
0
$$$$
0
$$$$
0
$$

3

2

$$
1
$$$$
31
$$$$
31
$$

7

8$$
6
$$$$
6
$$$$
0
$$$$
0
$$$$
0
$$$$
\begin{aligned}
& 0 \\
& 3
\end{aligned}
$$

3
13

$\begin{array}{ll}\% & \begin{array}{l}\text { Third } \\ \text { round }\end{array} \\ 0 & \end{array}$
$\% \quad \% \quad$ Fourth $\quad \% \quad \%$ diff. round diff.

$\begin{array}{llll}10 & 3 & 5.9 & -4.1\end{array}$

$\begin{array}{lllll}22 & 3 & 5.9 & -16.1 & 4\end{array}$

$\begin{array}{llll}38 & 22 & 43.1 & 5.1\end{array}$

$\begin{array}{lllll}14 & 7 & 13.7 & -0.3\end{array}$

$22 \quad 16$

70

$32 \quad 31$

$40 \quad 25$

8

$\begin{array}{llll}18 & 8 & 15.7 & -2.3\end{array}$

$\begin{array}{llll}12 & 3 & 5.9 & -6.1\end{array}$

2

$$
6
$$$$
4
$$$$
2
$$$$
\begin{array}{llll}
62 & 25 & 49.0 & -13
\end{array}
$$$$
\begin{array}{llll}
14 & 9 & 17.6 \quad 3.6
\end{array}
$$$$
\begin{array}{llll}
16 & 11 & 21.6 \quad 5.6
\end{array}
$$$$
\begin{array}{llll}
12 & 3 & 5.9 & -6.1
\end{array}
$$$$
\begin{array}{llll}
14 & 9 & 17.6 \quad 3.6
\end{array}
$$

$\begin{array}{lll}60.8 & 28.8 \quad 29\end{array}$

$74.4 \quad 13.6$

49.09

$17.6 \quad 3.6$

$$
6
$$$$
\begin{array}{llll}
26 & 10 & 19.6 & -6.4
\end{array}
$$

$\begin{array}{llll}38 & 21 & 41.2 & 3.2\end{array}$

8

$\begin{array}{llll}12 & 5 & 9.8 & -2.2\end{array}$

$\begin{array}{llll}10 & 7 & 13.7 & 3.7\end{array}$

$\begin{array}{llll}10 & 8 & 15.7 & 5.7\end{array}$

$\begin{array}{lllllll}64 & 21 & 41.2 & -22.8 & 17 & 43.6 & 2.4\end{array}$ 
Table 2 Mentioned factors (Continued)

living condition
access to ICT
products and technology for personal use in daily living
communication technology
personal devices (apps)
technological aids / means
means for physical impairments / access to assistive
devices
mobility aids
access to cognitive protheses
access to information and use of different channels
quality of instructions (easy to understand for MCI)
access to and dependence on transportation
accessibility / distance to public transport
accessibility / distance to (social) activities
accessibility / distance to facilities
country of residence
insurance policy of a country
official structured support / possibilities (e.g home care)
educational opportunity
availability and access to health care
policy

1
4
2
2
1
2
6

0

4

6

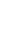

1

0

2

Personal factors

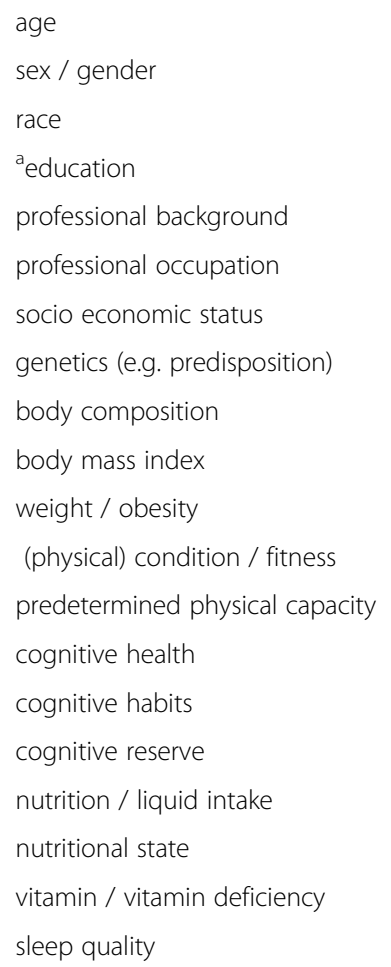

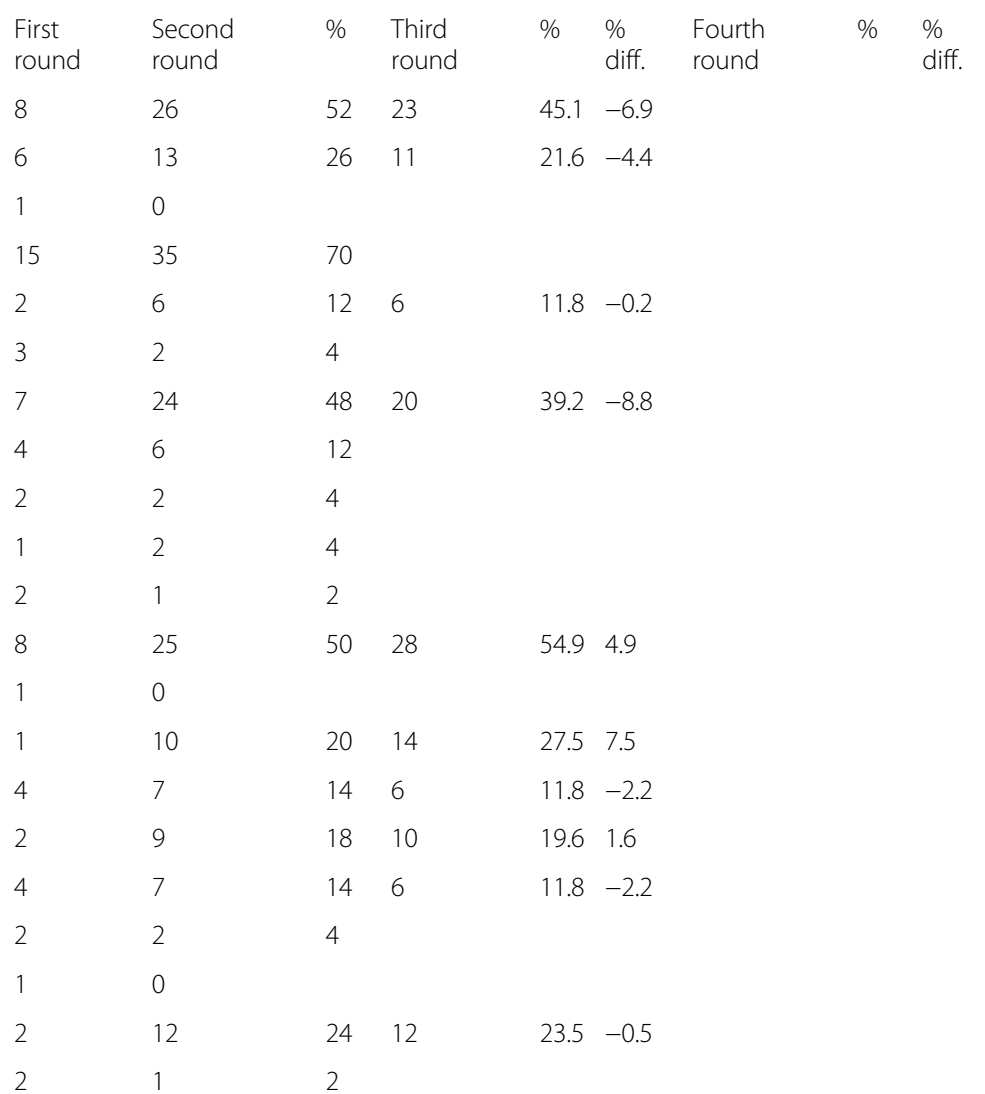


Table 2 Mentioned factors (Continued)

\begin{tabular}{|c|c|c|c|c|c|c|c|c|c|}
\hline balance between recreation and activity & 1 & 3 & 6 & & & & & & \\
\hline values & 2 & 5 & 10 & 3 & 5.9 & -4.1 & & & \\
\hline beliefs & 3 & 4 & 8 & & & & & & \\
\hline religion / spirituality & 5 & 5 & 10 & 2 & 3.9 & -6.1 & & & \\
\hline personal attitudes & 1 & 1 & 2 & & & & & & \\
\hline self-concept & 3 & 2 & 4 & & & & & & \\
\hline self-esteem & 2 & 2 & 4 & & & & & & \\
\hline self-satisfaction & 1 & 0 & & & & & & & \\
\hline self-efficacy & 4 & 13 & 26 & 16 & 31.4 & 5.4 & & & \\
\hline perceived stress & 2 & 2 & 4 & & & & & & \\
\hline well-being & 1 & 0 & & & & & & & \\
\hline sense of purpose in IADL tasks & 2 & 10 & 20 & 16 & 31.4 & 11.4 & & & \\
\hline personality & 10 & 22 & 44 & 20 & 39.2 & -4.8 & & & \\
\hline social skills & 7 & 8 & 16 & 10 & 19.6 & 3.6 & & & \\
\hline conation & 2 & 0 & & & & & & & \\
\hline desire for independence & 2 & 5 & 10 & 9 & 17.6 & 7.6 & & & \\
\hline behavior pattern & 3 & 0 & & & & & & & \\
\hline general initiative-taking & 1 & 2 & 4 & & & & & & \\
\hline extraversion & 2 & 0 & & & & & & & \\
\hline being open (e.g. willing to learn new things) & 3 & 5 & 10 & 3 & 5.9 & -4.1 & & & \\
\hline coping strategies & 8 & 25 & 50 & 28 & 54.9 & 4.9 & & & \\
\hline frustration tolerance & 1 & 0 & & & & & & & \\
\hline willing to ask for and accept someone's help & 3 & 8 & 16 & 13 & 25.5 & 9.5 & & & \\
\hline flexibility / creativity & 2 & 1 & 2 & & & & & & \\
\hline resilience & 3 & 8 & 16 & 5 & 9.8 & -6.2 & & & \\
\hline hobbies & 2 & 1 & 2 & & & & & & \\
\hline interests & 4 & 5 & 10 & 8 & 15.7 & 5.7 & & & \\
\hline maintenance of habits (e.g. hobbies, interests, sexuality) & 1 & 6 & 12 & 8 & 15.7 & 3.7 & & & \\
\hline personal hygiene & 3 & 0 & & & & & & & \\
\hline personal habits (e.g. not have done certain IADL lifelong) & 1 & 8 & 16 & 13 & 25.5 & 9.5 & & & \\
\hline personal routine & 3 & 3 & 6 & & & & & & \\
\hline personal (daily) structure & 2 & 2 & 4 & & & & & & \\
\hline physical activity (past and current) & 6 & 15 & 30 & 15 & 29.4 & -0.6 & & & \\
\hline enjoy of physical activity & 2 & 0 & & & & & & & \\
\hline social activities & 2 & 3 & 6 & & & & & & \\
\hline moral conduct & 3 & 0 & & & & & & & \\
\hline family position & 2 & 1 & 2 & & & & & & \\
\hline (gender) roles & 4 & 0 & & & & & & & \\
\hline social integration / connectedness & 8 & 15 & 30 & 18 & 35.3 & 5.3 & & & \\
\hline socio-cultural background & 13 & 10 & 20 & 10 & 19.6 & -0.4 & & & \\
\hline upbringing & 3 & 0 & & & & & & & \\
\hline literacy / health literacy & 1 & 3 & 6 & & & & & & \\
\hline experience / biography & 7 & 5 & 10 & 4 & 7.8 & -2.2 & & & \\
\hline experience - (e.g. familiarity with certain IADL tasks) & 6 & 10 & 20 & 21 & 41.2 & 21.2 & 23 & 59 & 17.8 \\
\hline computer literacy & 3 & 0 & & & & & & & \\
\hline
\end{tabular}


Table 2 Mentioned factors (Continued)

\begin{tabular}{|c|c|c|c|c|c|c|}
\hline physical health & 6 & 7 & 14 & 8 & 15.7 & 1.7 \\
\hline neurological medical conditions & 3 & 4 & 8 & & & \\
\hline musculoskeletal medical conditions & 5 & 3 & 6 & & & \\
\hline treatment of physical illness & 1 & 0 & & & & \\
\hline multimorbidity & 1 & 3 & 6 & & & \\
\hline comorbidities & 3 & 8 & 16 & 12 & 23.5 & 7.5 \\
\hline disease duration & 1 & 0 & & & & \\
\hline frailty & 5 & 6 & 12 & 12 & 23.5 & 11.5 \\
\hline psychological health & 8 & 17 & 34 & 23 & 45.1 & 11.1 \\
\hline psychosis & 2 & 0 & & & & \\
\hline depression & 13 & 17 & 34 & 20 & 39.2 & 5.2 \\
\hline anxiety & 9 & 4 & 8 & & & \\
\hline current medication / possible side effects & 5 & 3 & 6 & & & \\
\hline smoking & 3 & 1 & 2 & & & \\
\hline alcohol consumption & 2 & 2 & 4 & & & \\
\hline addiction / substance misuse & 5 & 2 & 4 & & & \\
\hline
\end{tabular}

Factors mentioned in the first round, frequency, \%: percent-agreement between panelists; \%-diff: difference in percent-agreement between two succeeding rounds - a positive number indicates more agreement

${ }^{\text {a }}$ actors included after second round

$b_{\text {factors included after third round }}$

c factors included after fourth round

Table 3 Critical comments on the model

\begin{tabular}{ll}
\hline${ }^{a}$ Categories & Frequency (percent) \\
\hline Same weighting for all factors & $4(12 \%)$ \\
Balance as separate factor from mobility & $4(12 \%)$ \\
Mental health not included & $4(12 \%)$ \\
Relatedness of factors not included & $3(9 \%)$ \\
$\begin{array}{l}\text { Problem solving / planning included in executive } \\
\text { functions }\end{array}$ & $3(9 \%)$ \\
Environmental factors - products and technology & $3(9 \%)$ \\
not included & \\
Environmental factors - natural environment not & $2(6 \%)$ \\
included & \\
$\begin{array}{l}\text { Vision / hearing functions not gathered as sensory } \\
\text { functions }\end{array}$ & $2(6 \%)$ \\
$\begin{array}{l}\text { Physical function factors (others than balance) } \\
\text { not included }\end{array}$ & $2(6 \%)$ \\
Visuospatial functions not included & $1(3 \%)$ \\
Language functions not included & $1(3 \%)$ \\
Fine motor skills not included & $1(3 \%)$ \\
Motivation not included & $1(3 \%)$ \\
Method not appropriate & $1(3 \%)$ \\
\hline
\end{tabular}

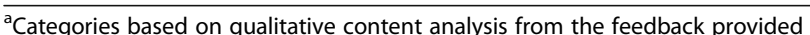
on the model environmental factors, that are thought to influence IADL performance in people with MCI.

To our knowledge, this study is the first to propose a comprehensive model on the influencing factors on IADL performance in people with $\mathrm{MCI}$, incorporating all domains of the ICF framework. IADL performance in people with MCI is highly individual and might be dependent on the culture and environment a person lives in, which was represented by the wide variety of factors mentioned in the first round of the Delphi survey. Although the Delphi method included relevant researchers and clinicians, the model may not be conclusive. However, a substantial number of the factors reaching consensus are consistent with the findings from empirical data [17-20, 24-29, 40, 41], while others have been neglected in the literature so far, e.g. functional mobility. Thus, our model might provide a better understanding of IADL functioning in people with MCI and serve as a ground for future research. Cross-sectional or cohort studies on IADL functioning might use the model as a base to decide which factors should be investigated; intervention studies might use our model as theoretical background in the development of novel interventions that aim to improve IADL functioning in people with MCI. Nonetheless, our model might have implications for clinical practice by strengthening the awareness that IADL functioning is influenced not merely by cognition. Considering all factors in the treatment of people with MCI with IADL impairments might 


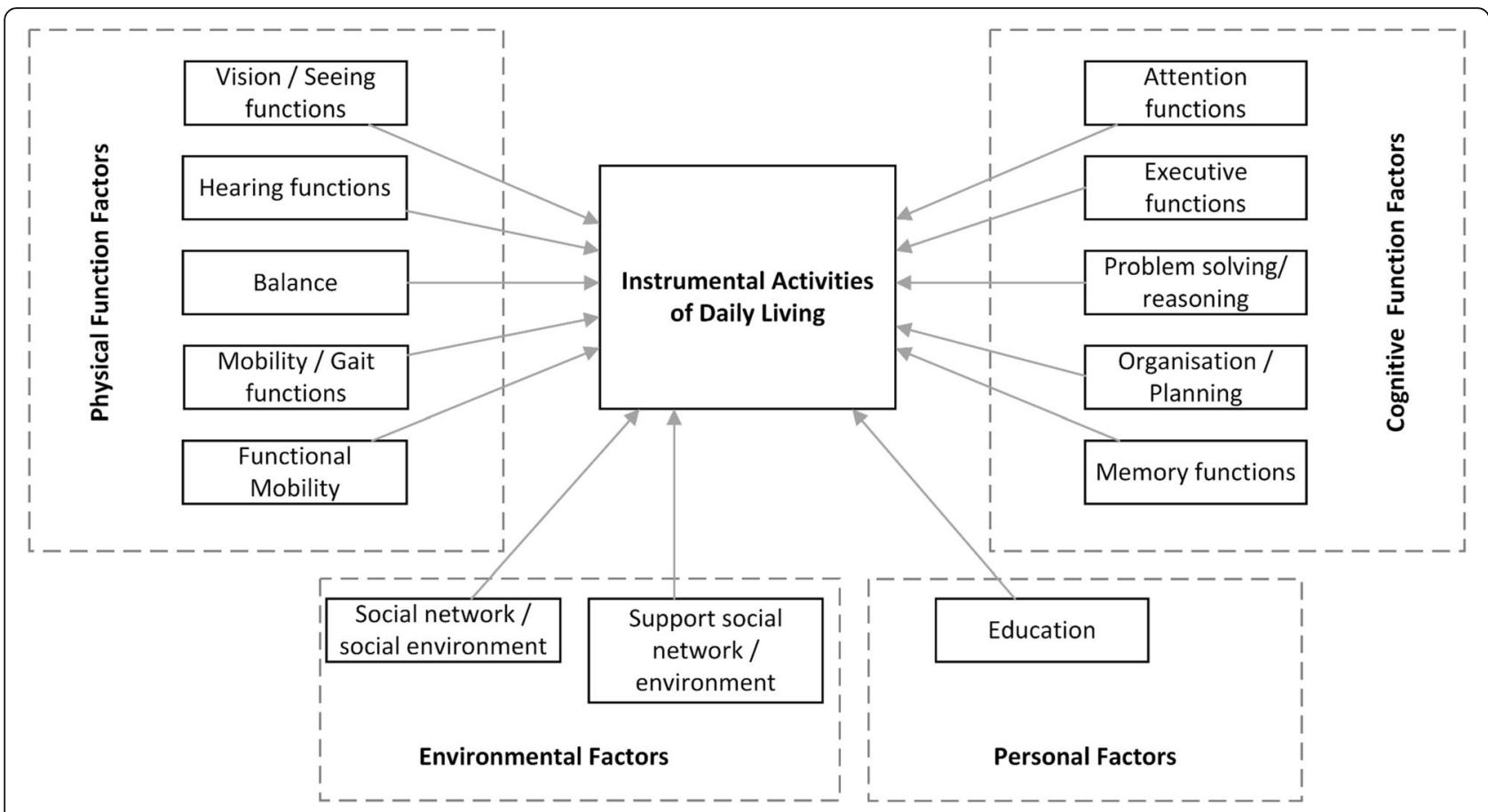

Fig. 1 Model of IADL functioning in people with $\mathrm{MCI}$

help to improve their level of functioning; for instance, by counteracting impaired sensory functions with an appropriate aid. Additionally, our model might have an impact on the way IADL functioning is assessed.

\section{Cognitive function factors}

Multiple cognitive function factors were included in the model. Consensus was reached for memory, attention and executive function, as well as executive function subdomains organization / planning and problem solving / reasoning. The bulk of literature investigating the question of which cognitive domains account for IADL performance is not consistent. Despite the widely accepted assumption that IADL performance is mainly affected by cognition, Royall et al. suggest that, based on empirical data, less than $8 \%$ of IADL variance is explained by cognition [42]. Furthermore, in another study, the same group ascertained in their empirically- based model that intelligence accounts for at least $50 \%$ of the variance in IADL performance in people with MCI [43]. The fact that IADL performance is independent of cognitive performance measures and the fraction of intelligence is related to IADL, may both serve as a dementia severity metric [43]. However, in our study intelligence did not reach consensus and our results contradict the findings of empirical studies. On the other hand, in their meta-analysis, McAlister et al. revealed that cognitive functions accounted for $23 \%$ of the variability in IADL performance in people with MCI [17].
Among the cognitive domains, executive function (37\%), attention (33\%) and memory (23\%) explained a certain amount of variance in IADL performance, while planning / organization and problem solving / reasoning explained a smaller amount of variance [17]. In our study, the subdomains planning / organization and problem solving / reasoning were explicitly raised by the panel and reached consensus in addition to executive function. One may argue, that the subdomains are already encompassed by executive function. However, executive function is an umbrella term [44] and heterogeneous definitions of subdomains are found in the literature [17, 45]. Therefore, we did not exclude the subdomains from the model. Furthermore, an exclusion of these subdomains would have contradicted our predefined cut-offs regarding consensus. The meta-analysis by McAlister et al. also detected other cognitive domains and executive function subdomains not included or explicitly mentioned in our model (e.g. switching, judgment / decision making and working memory) that explained a remarkable amount of variance [17]. Therefore, factors that did not reach consensus in our Delphi process, but that showed a remarkable amount of agreement, might still be added to the model in future studies, e.g. language functions $(67 \%)$.

\section{Physical function factors}

Certain IADL tasks need appropriate sensory functions. Not surprisingly, visual and hearing functions were 
included in the model in accordance with the current literature. A longitudinal study indicated that visual and hearing impairments are related to self-reported functional impairments in old people [24]. Furthermore, sensory restrictions are associated with slight IADL changes [27] and the presence of visual and hearing impairment in combination with cognitive decline was associated with impaired IADL performance in older adults [25].

Balance was included in the model even though some panelists suggested that balance is a subdomain of gait functions. Impaired balance does have an impact on gait function, but several IADL tasks also require static balance abilities [46]. For this reason, balance was not summarized under gait functions. Literature on balance in people with MCI is sparse. However, studies using instrumented assessments did find impaired balance functions in people with MCI [20]. Moreover, studies using clinical assessments of balance, e.g. POMA, revealed an association between IADL performance and balance in people with MCI [26].

Mobility / gait functions were included in the model, which is supported by the current literature [20]. Different aspects of gait function were found to be impaired in people with MCI [19, 47-49]. A remarkable number of IADLs require sound gait functions, e.g. doing the shopping or using public transport.

A further factor included in the model was functional mobility, e.g. walking stairs or functional reach, although functional mobility related to IADL performance has had little attention in literature to date. Therefore, future studies investigating IADL performance in people with MCI should consider functional mobility as a possible influencing factor.

Physical function factors that might affect IADL performance [40], e.g. muscle power functions, reached a remarkable percent-agreement (65\%), but insufficient consensus to be included in the model. Mobility/gait functions and functional mobility presume, inter alia, appropriate muscle power functions. In addition, grip strength may be associated with functional impairments in people with MCI [23]. Therefore, the factor muscle power functions might be worth considering in studies investigating the influence of physical function factors on IADL performance in people with MCI.

\section{Environmental factors}

Based on the panelists' suggestions, "Network / Social Environment" and "Network / Social Environment Support" were included in the model. Intervention studies including study partners reported positive findings on IADL performance in people with MCI [31,32], leading to the conclusion that these factors play an important role.

Several environmental factors were mentioned in the first round but failed to reach consensus. Some were also raised during the feedback on the model: natural environment, e.g. place of residence, housing and products and technology (technical aids). The importance of compensatory strategies and use of technical aids in the performance of IADL in people with $\mathrm{MCI}$ has been highlighted in literature [30]. Furthermore, these factors underline the individuality of IADL functioning and might be considered in the design of future studies or interventions on IADL performance in people with MCI.

\section{Personal factors}

The only personal factor included in the model was education. Education and cognitive function might be related in people with MCI. Education is usually included as a possible confounder in empirical studies. However, conclusions from literature are not clear. In a longitudinal study on a sample of Asian older adults, lower education was associated with greater IADL dependence [28], while a higher level of cognitive reserve delayed the onset of cognitive decline [29]. In contrast, a metaanalysis did not find education as a mediator of the relationship between cognitive function and IADL [17].

Literature suggests additional personal factors that might influence IADL performance in MCI [3] but with inconsistent findings. Age was found to be associated with impaired IADL performance in MCI [28, 50], as well as depression $[28,51]$, frailty [26], physical activity [52] and comorbidities [28, 53]. In contrast, Mariani and colleagues revealed that IADL performance was more strongly related to cognitive function than physical comorbidities [27]. The inconclusive findings in literature, as well as the ratings in the Delphi process, underline the individual nature of IADL performance in people with MCI.

\section{Strength and limitations}

One strength of our study is the number of panelists, with half of them working in research and academia and the other half in clinical settings. The great amount of experience of the panelists in the field of MCI and IADL performance is also noteworthy. Unfortunately, the panel did not include experts from the Asian or African continents due to non-response and we consequently do not know if and how African or Asian panelists would have influenced the model. The response rates in the first three rounds of the Delphi survey were very high. Another strength of this study is that it used a different approach to modelling IADL functioning in people with $\mathrm{MCI}$ and the new insights could provide a basis for future research.

This study also has several limitations. A Delphi study reports only the results from a consensus of expert opinions on a topic and could contradict findings from empirical studies [38]. Performing a systematic review 
would have been a different approach to investigate the possible contributing factors on IADL performance in MCI. However, systematic reviews performed in this field have faced similar problems: the constructs of interest (i.e. $\mathrm{MCI}, \mathrm{IADL}$ ) have been defined and operationalized in different ways $[5,7,12,17,54]$. Furthermore, the type and number of assessments used to measure the outcomes of interest were heterogeneous $[5,7,12,17,54]$. Moreover, the results are limited to the factors investigated in the included studies and might not be fully encompassing. Comparable problems would arise from empirical studies: a retrospective analysis of pre-existing data sets would be limited to the outcomes assessed; in a prospective design, it remains unclear which factors should be assessed, given the huge range of possibilities, e.g. domains provided by the ICF. Thus, we took a deductive approach to build a model (theoretical) based on the panelists great insight and understanding of IADL functioning in people with $\mathrm{MCI}$; we suggest our model should be used as a starting point for further elaboration based on an inductive approach using empirical data.

The definition of consensus in Delphi studies is somewhat arbitrary [34]. One might argue, that the predefined cut-off level of $\geq 70 \%$ percent-agreement for factors to be included in the model was set too low. However, this study included a heterogeneous sample of panelists and, therefore, very high percent-agreements were not anticipated. Alternatively, the cut-off might have been set too high resulting in relevant factors with substantial percentagreement being excluded from the model. These might be considered in future studies, as previously discussed.

Finally, due to the design of our study, it was not possible to weight the factors. In the feedback round some panelists pointed out that some factors are more important than others. Therefore, weighting of the factors in general and across different cultures should be incorporated in future studies investigating IADL performance in people with $\mathrm{MCI}$.

\section{Conclusion}

The results of this study suggest that IADL performance in people with MCI is affected not only by cognitive function factors, but also by various physical function factors, personal factors and environmental factors. Therefore, it is crucial to consider all these factors in future studies in people with MCI exploring IADL performance, as well as in the design and investigation of new interventions to improve everyday activities. Finally, our results may have implications for clinical practice in people with MCI, both in the methods of assessing IADLs and the treatment of IADL impairments.

\section{Supplementary information}

Supplementary information accompanies this paper at https://doi.org/10. 1186/s12883-020-01843-9.

Additional file 1. First-round Questionnaire. The file contains the questionnaire from the first round; it was downloaded from EFS survey on 23. September 2019: https://ww2.unipark.de/www/print_survey.php?syid=515 897\&menu_node=print 2 .

\section{Abbreviations}

IADL: Instrumental Activities of Daily Living; ICF: International Classification of Functioning, Disability and Health; MCl: Mild Cognitive Impairment; POMA: Performance Oriented Mobility Assessment

\section{Acknowledgements}

The authors give their thanks to all the panelists for their participation and for sharing their thoughts and suggestions; Ms. Riana Saba for her contribution in the categorization of factors; Mrs. Sabrina Stängle-Fehn for her support in implementing the questionnaires in the EFS survey; and the colleagues of the research and development team from the Institute of Physiotherapy at Zurich University of Applied Sciences for pretesting the questionnaires.

\section{Authors' contributions}

MB participated in the study design and conception, data acquisition and analysis, wrote the first draft of the manuscript and revised new drafts. SAMS and TM participated in the study design and conception and manuscript drafting. KN participated in the study design and conception, data analysis and revised new drafts of the manuscript. All authors read and approved the final submitted manuscript.

\section{Funding}

This work did not receive funding.

\section{Availability of data and materials}

The datasets generated and analyzed during the study are available upon reasonable request to the corresponding author.

\section{Ethics approval and consent to participate}

In accordance with the Swiss regulations on research involving humans this study does not fall within the scope of an ethical approval (Cantonal Ethics Committee Zurich; No: Req-2019-00110). The request to the Ethics Committee included a detailed description of the consent and data collection procedures. Panelists were invited and gave their written informed consent to participate in the Delphi study by email. Additionally, each rounds' questionnaire included a consent form. In accordance with the European General Data Protection Regulation, the consent form included all necessary information, and all panelists explicitly gave their consent for the use of their data for research purposes. To participate in the survey, panelists had to click on a button to consent with the data collection before filling out the questionnaires.

\section{Consent for publication}

Not applicable.

\section{Competing interests}

All authors disclose any competing interests.

\section{Author details}

${ }^{1}$ School of Helath Professions, Institute of Physiotherapy, Zurich University of Applied Sciences, Winterthur, Switzerland. ${ }^{2}$ Department of Health Sciences and Health Policy, University of Lucerne, Lucerne, Switzerland. ${ }^{3}$ Alzheimer Center Amsterdam, Amsterdam University Medical Centers / Department of Clinical, Neuro and Developmental Psychology, VU University / Amsterdam Neuroscience, Amsterdam, the Netherlands. ${ }^{4}$ Geriatrische Klinik St.Gallen, St.Gallen, Switzerland. ${ }^{5}$ Department of Geriatrics and Aging Research, University Hospital and University of Zurich, Zurich, Switzerland. 
Received: 25 November 2019 Accepted: 24 June 2020 Published online: 01 July 2020

\section{References}

1. Lawton MP, Brody EM. Assessment of older people: self-maintaining and instrumental activities of daily living. Gerontologist. 1969;9:179-86.

2. Sikkes SA, Rotrou J. A qualitative review of instrumental activities of daily living in dementia: what's cooking? Neurodegener Dis Manag. 2014;4:393400.

3. Gold DA. An examination of instrumental activities of daily living assessment in older adults and mild cognitive impairment. J Clin Exp Neuropsychol. 2012;34:11-34.

4. Heyn PC, Johnson KE, Kramer AF. Endurance and strength training outcomes on cognitively impaired and cognitively intact older adults: a meta-analysis. J Nutr Health Aging. 2008;12:401-9.

5. Giebel CM, Challis D, Montaldi D. Understanding the cognitive underpinnings of functional impairments in early dementia: a review. Aging Ment Health. 2015;19:859-75.

6. Reed C, Belger M, Vellas B, Andrews JS, Argimon JM, Bruno G, et al. Identifying factors of activities of daily living important for cost and caregiver outcomes in Alzheimer's disease. Int Psychogeriatr. 2016;28:24759.

7. Royall DR, Lauterbach EC, Kaufer D, Malloy P, Coburn KL, Black KJ, et al. The cognitive correlates of functional status: a review from the committee on research of the American neuropsychiatric association. J Neuropsychiatry Clin Neurosci. 2007;19:249-65.

8. Reppermund S, Sachdev PS, Crawford J, Kochan NA, Slavin MJ, Kang K, et al. The relationship of neuropsychological function to instrumental activities of daily living in mild cognitive impairment. Int J Geriatr Psychiatry. 2011;26: 843-52.

9. Marshall GA, Amariglio RE, Sperling RA, Rentz DM. Activities of daily living: where do they fit in the diagnosis of Alzheimer's disease? Neurodegener Dis Manag. 2012;2:483-91.

10. Winblad B, Palmer K, Kivipelto M, Jelic V, Fratiglioni L, Wahlund LO, et al. Mild cognitive impairment--beyond controversies, towards a consensus: report of the international working group on mild cognitive impairment. J Intern Med. 2004:256:240-6.

11. Jekel K, Damian M, Wattmo C, Hausner L, Bullock R, Connelly PJ, et al. Mild cognitive impairment and deficits in instrumental activities of daily living: systematic review. Alzheimers Res Ther. 2015;7(1):17.

12. Lindbergh CA, Dishman RK, Miller LS. Functional disability in mild cognitive impairment: a systematic review and meta-analysis. Neuropsychol Rev. 2016; 26:129-59.

13. Rodakowski J, Skidmore ER, Reynolds CF 3rd, Dew MA, Butters MA, Holm MB, et al. Can performance on daily activities discriminate between older adults with normal cognitive function and those with mild cognitive impairment? J Am Geriatr Soc. 2014;62:1347-52.

14. Sikkes SA, Visser PJ, Knol DL, de Lange-de Klerk ES, Tsolaki M, Frisoni GB, et al. Do instrumental activities of daily living predict dementia at 1- and 2year follow-up? Findings from the development of screening guidelines and diagnostic criteria for Predementia Alzheimer's disease study. J Am Geriatr Soc. 2011;59:2273-81.

15. Farias ST, Lau K, Harvey D, Denny KG, Barba C, Mefford AN. Early functional limitations in cognitively Normal older adults predict diagnostic conversion to mild cognitive impairment. J Am Geriatr Soc. 2017;65:1152-8.

16. Sachdev PS, Blacker D, Blazer DG, Ganguli M, Jeste DV, Paulsen JS, et al. Classifying neurocognitive disorders: the DSM-5 approach. Nat Rev Neurol. 2014;10:634-42

17. McAlister C, Schmitter-Edgecombe M, Lamb R. Examination of variables that may affect the relationship between cognition and functional status in individuals with mild cognitive impairment: a meta-analysis. Arch Clin Neuropsychol. 2016;31:123-47.

18. Beauchet $\mathrm{O}$, Allali G, Montero-Odasso M, Sejdic E, Fantino B, Annweiler C. Motor phenotype of decline in cognitive performance among communitydwellers without dementia: population-based study and meta-analysis. PLoS One. 2014;9:6.

19. Montero-Odasso M, Oteng-Amoako A, Speechley M, Gopaul K, Beauchet O, Annweiler $C$, et al. The motor signature of mild cognitive impairment: results from the gait and brain study. J Gerontol A Biol Sci Med Sci. 2014;69: $1415-21$.
20. Bahureksa L, Najafi B, Saleh A, Sabbagh M, Coon D, Mohler MJ, et al. The impact of mild cognitive impairment on gait and balance: a systematic review and meta-analysis of studies using instrumented assessment. Gerontology. 2017:63:67-83.

21. Bolandzadeh N, Kording K, Salowitz N, Davis JC, Hsu L, Chan A, et al. Predicting cognitive function from clinical measures of physical function and health status in older adults. PLoS One. 2015;10:3.

22. World Health Organization. International Classification of Functioning, Disability and Health (ICF). Geneva: World Health Organization (WHO); 2001. http://www.who.int/classifications/icf/en/. Accessed 21 Oct 2019.

23. Vermeulen J, Neyens JC, van Rossum E, Spreeuwenberg MD, de Witte LP. Predicting ADL disability in community-dwelling elderly people using physical frailty indicators: a systematic review. BMC Geriatr. 2011;11:33.

24. Brennan M, Su YP, Horowitz A. Longitudinal associations between dual sensory impairment and everyday competence among older adults. J Rehabil Res Dev. 2006:43:777-92.

25. Guthrie DM, Davidson JGS, Williams N, Campos J, Hunter K, Mick P, et al. Combined impairments in vision, hearing and cognition are associated with greater levels of functional and communication difficulties than cognitive impairment alone: analysis of interRAl data for home care and long-term care recipients in Ontario. PLoS One. 2018;13:2.

26. Nyunt MSZ, Soh CY, Gao Q, Gwee X, Ling ASL, Lim WS, et al. Characterisation of Physical Frailty and Associated Physical and Functional Impairments in Mild Cognitive Impairment. Front Med (Lausanne). 2017;4:230.

27. Mariani E, Monastero R, Ercolani S, Rinaldi P, Mangialasche F, Costanzi E et al. Influence of comorbidity and cognitive status on instrumental activities of daily living in amnestic mild cognitive impairment: results from the ReGAI project. Int J Geriatr Psychiatry. 2008;23:523-30.

28. Ng TP, Niti M, Chiam PC, Kua EH. Physical and cognitive domains of the instrumental activities of daily living: validation in a multiethnic population of Asian older adults. J Gerontol A Biol Sci Med Sci. 2006;61:726-35.

29. Soldan A, Pettigrew C, Cai Q, Wang J, Wang MC, Moghekar A, et al. Cognitive reserve and long-term change in cognition in aging and preclinical Alzheimer's disease. Neurobiol Aging. 2017;60:164-72.

30. Schmitter-Edgecombe M, Parsey C, Lamb R. Development and psychometric properties of the instrumental activities of daily living: compensation scale. Arch Clin Neuropsychol. 2014;29:776-92.

31. Schmitter-Edgecombe M, Dyck DG. Cognitive rehabilitation multi-family group intervention for individuals with mild cognitive impairment and their care-partners. J Int Neuropsychol Soc. 2014;20:897-908.

32. Greenaway M, Duncan N, Smith G. The memory support system for mild cognitive impairment: randomized trial of a cognitive rehabilitation intervention. Int J Geriatr Psychiatry. 2013;28:402-9.

33. Hasson F, Keeney S, McKenna H. Research guidelines for the Delphi survey technique. J Adv Nurs. 2000;32:1008-15.

34. von der Gracht HA. Consensus measurement in Delphi studies. Technol Forecast Soc Change. 2012;79:1525-36.

35. Diamond IR, Grant RC, Feldman BM, Pencharz PB, Ling SC, Moore AM, et al. Defining consensus: a systematic review recommends methodologic criteria for reporting of Delphi studies. J Clin Epidemiol. 2014;67:401-9.

36. Trevelyan EG, Robinson PN. Delphi methodology in health research: how to do it? Eur J Integr Med. 2015;7:423-8.

37. Prinsen CA, Vohra S, Rose MR, King-Jones S, Ishaque S, Bhaloo Z, et al. Core Outcome Measures in Effectiveness Trials (COMET) initiative: protocol for an international Delphi study to achieve consensus on how to select outcome measurement instruments for outcomes included in a 'core outcome set'. Trials. 2014;15:247.

38. De Vet E, Brug J, De Nooijer J, Dijkstra A, De Vries NK. Determinants of forward stage transitions: a Delphi study. Health Educ Res. 2005;20:195-205.

39. Mayring P. Qualitative Inhaltsanalyse Grundlagen und Techniken. 7th ed. Deutscher Studienverlag: Weinheim; 2000

40. Aggarwal NT, Wilson RS, Beck TL, Bienias JL, Bennett DA. Motor dysfunction in mild cognitive impairment and the risk of incident Alzheimer disease. Arch Neurol. 2006:63:1763-9.

41. Schmitter-Edgecombe M, Parsey CM. Cognitive correlates of functional abilities in individuals with mild cognitive impairment: comparison of questionnaire, direct observation, and performance-based measures. Clin Neuropsychol. 2014:28:726-46.

42. Royall DR, Palmer RF, O'Bryant SE, Texas Alzheimer's R, Care C. Validation of a latent variable representing the dementing process. J Alzheimers Dis. 2012;30:639-49. 
43. Royall DR, Palmer RF. Getting past "g": testing a new model of dementing processes in persons without dementia. J Neuropsychiatry Clin Neurosci. 2012;24:37-46.

44. Diamond A. Executive Functions. Annu Rev Psychol. 2013;64:135-68.

45. Sachdev PS, Mohan A, Taylor L, Jeste DV. DSM-5 and Mental Disorders in Older Individuals: An Overview. Harv Rev Psychiatry. 2015;23:320-8.

46. Horak FB. Postural orientation and equilibrium: what do we need to know about neural control of balance to prevent falls? Age Ageing. 2006;35(Suppl 2):ii7-ii1 1.

47. Verghese J, Robbins M, Holtzer R, Zimmerman M, Wang C, Xue X, et al. Gait dysfunction in mild cognitive impairment syndromes. J Am Geriatr Soc. 2008:56:1244-51

48. Beauchet O, Allali G, Launay C, Herrmann FR, Annweiler C. Gait variability at fast-pace walking speed: a biomarker of mild cognitive impairment? J Nutr Health Aging. 2013:17:235-9.

49. Muir SW, Speechley M, Wells J, Borrie M, Gopaul K, Montero-Odasso M. Gait assessment in mild cognitive impairment and Alzheimer's disease: the effect of dual-task challenges across the cognitive spectrum. Gait Posture. 2012;35: 96-100.

50. Artero S, Touchon J, Ritchie K. Disability and mild cognitive impairment: a longitudinal population-based study. Int J Geriatr Psychiatry. 2001;16:1092-7.

51. Cahn DA, Malloy PF, Salloway S, Rogg J, Gillard E, Kohn R, et al. Subcortical hyperintensities on MRI and activities of daily living in geriatric depression. J Neuropsychiatry Clin Neurosci. 1996:8:404-11.

52. Storeng SH, Sund ER, Krokstad S. Factors associated with basic and instrumental activities of daily living in elderly participants of a populationbased survey: the Nord-Trondelag health study, Norway. BMJ Open. 2018;8: 3.

53. Wang L, van Belle G, Kukull WB, Larson EB. Predictors of functional change: a longitudinal study of nondemented people aged 65 and older. J Am Geriatr Soc. 2002;50:1525-34.

54. Nygard L. Instrumental activities of daily living: a stepping-stone towards Alzheimer's disease diagnosis in subjects with mild cognitive impairment? Acta Neurol Scand. 2003:Suppl 179:42-6.

\section{Publisher's Note}

Springer Nature remains neutral with regard to jurisdictional claims in published maps and institutional affiliations.

Ready to submit your research? Choose BMC and benefit from:

- fast, convenient online submission

- thorough peer review by experienced researchers in your field

- rapid publication on acceptance

- support for research data, including large and complex data types

- gold Open Access which fosters wider collaboration and increased citations

- maximum visibility for your research: over $100 \mathrm{M}$ website views per year

At $\mathrm{BMC}$, research is always in progress.

Learn more biomedcentral.com/submissions 